\title{
Generating route instructions with varying levels of detail
}

\author{
Jürgen Ziegler, Tim Hussein, Daniel Münter, Jens Hofmann, Timm Linder \\ University of Duisburg-Essen \\ Lotharstr. 65, 47057 Duisburg, Germany \\ \{firstname.lastname\}@uni-due.de
}

\begin{abstract}
In this paper, we present a technique for adaptive generation of personalized route instructions based on the driver's knowledge of particular route sections. We evaluated the mechanism with two empirical studies, both attesting significant preference for the adaptively generated presentations over an established online service (Google Maps).
\end{abstract}

\section{Categories and Subject Descriptors}

H.3.4 [Information Storage and Retrieval]: Systems and Software-Current awareness systems (selective dissemination of information-SDI); H.3.5 [Information Storage and Retrieval]: Online Information Services-Commercial services, Web-based services

\section{General Terms}

Design, Framework, Implementation

\section{Keywords}

Automotive, navigation systems, usability

\section{INTRODUCTION}

Navigation systems have become a common accessory in current automobiles. With regard to the preciseness and reliability of traffic routing $[18,5]$ as well as the overall usability of navigation systems [9], considerable progress has been made over the past years. With respect to optimizing the trade-off between providing supportive and reliable guidance and minimizing the level of driver disruption, however, there is still room for improvement. A major shortcoming of current navigation systems can be seen in the fact that they do not take the driver's personal knowledge into account. Most existing systems are not capable of personalizing the directions given depending on the user's familiarity with the route to be taken or other geospatial knowledge. Therefore, directions are mostly presented in a very detailed, turn-byturn fashion, even when the driver knows the area very well, oftentimes unnecessarily distracting or annoying the driver.

Submitted to Automotive UI 2011
Several authors have pointed at the relation between the user's knowledge and routing instructions. Lovelace et al. showed that the importance of explicitly mentioning the different sections of a route decreases with familiarity of the area [10]. On well-known parts of a route, detailed turn-byturn directions are not necessary [13]. Forlizzi et al. showed that persons were directing their attention to the navigation process only in unknown areas [4]. A recent study identified a personalized presentation of routes as a key requirement for more effective navigation systems [1]. Present solutions, however, are not personalized and thus do not take the driver's personal knowledge, experience, and preferences into account. Our assumption is that incorporating a personalization mechanism in navigation systems can lead to an improved overall driving experience with reduced disruption and annoyance on the part of the driver. In such an approach, instructions should be rather concise and coarsegrained when the user knows the particular area well, and more detailed when driving through unknown territory.

In this paper, we address this problem by an approach transforming conventional route directions into a layered model of directions, where each layer reflects a different level of granularity. Applications using such a model can adapt the directives to the driver's preferences and knowledge of the particular area in order to display only necessary and helpful information instead of every single detail.

For that purpose, we implemented a technique that uses semantically enriched models of itineraries to create variations of the directions presented with different granularity (Section 3). To obtain evidence for the appropriateness of the approach and to determine suitable levels of detail, we carried out two user studies, in which we could show that users prefer personalized routes generated by the system over conventional route descriptions generated by Google Maps (Section 4). The article concludes with a discussion and a a brief outlook (Section 5).

\section{RELATED WORK}

The way humans formulate route descriptions is strongly dependent on the individual's mental model of the spatial surroundings of a route and its start and end points [17]. Depending on the spatial knowledge that is present in this model, route descriptions may consist of different types of instructions. The instructions might, for instance, refer to popular places or personally known places ("near aunt Mary's house"), compass directions, or simply provide a sequential 
description of the required changes in direction. Today's navigation systems only use a very limited range of these concepts and most systems present their instructions always at the same, fixed level of detail (LOD).

\section{Route Descriptions}

Driving instructions that notify the user of impending lane or direction changes at decision points are called "turn-byturn directions". They are especially helpful if the driver has no prior spatial knowledge of the area at all. However, this assumption is usually only valid for a limited part of the route, since most routes tend to either start or end in a familiar area, such as the place of work or residence.

Moreover, pure turn-by-turn instructions do not coincide with the way humans intuitively describe complex routes. In route descriptions that are personally communicated by humans, lots of unnecessary details are usually left out. Instead, spatial knowledge of the surroundings is activated, relevant information identified and structured, and then communicated in verbalized form [10]. During this process, several consecutive instructions are frequently combined into a more abstract, higher-level instruction. Klippel et al. refer to this technique as "spatial chunking" [8]. A simple example of this is the abstracted instruction "turn right at the third traffic light", which does not explicitly refer to the preceding two traffic lights. This concrete type of chunking is called numerical chunking, which refers to the aggregation of several objects of the same type. A different approach is chunking based upon landmarks, i.e. prominent spots alongside a route, for instance distinctive buildings or highways. A driving instruction that utilizes landmark-based chunking might be to "drive to highway 3" [7].

Yet another approach focuses on describing the destination of a route by utilizing places or building on the way. This might, for example, become useful to a taxi driver, who has generally got a good knowledge of the city, but might not know the name of every individual street. By providing the driver with the information that the destination street is located in a specific district in town, the taxi driver can utilize personal knowledge of where the different districts are located, even if the destination street itself is unknown. Here, advantage is taken of the hierarchical organization of spatial knowledge [15].

The approaches mentioned last are referred to as "destination descriptions" in the literature [16]. In summary, they state that persons familiar with a certain area can provide route descriptions by referring to well-known objects. Driving instructions of this kind tell the driver where to drive next, without going into too much detail about the how. Thus, there is a direct relationship between the driver's spatial knowledge of a given area, and the (required) level of driving instruction detail.

Providing appropriate abstractions therefore serves as an important means of reducing the complexity involved in the cognitive processing of spatial information [7]. It is, however, a factor which is currently not sufficiently being taken into account for computer-based generation of route descriptions: Current navigation systems confront the user with a new instruction at every possible decision point, which un- necessarily increases the driver's cognitive workload [3].

\section{Existing adaptation approaches}

With "MyRoute", Patel et al. [11] present an approach for route personalization that generates a condensed route description in two steps. First, landmarks the user knows well as well as routing information are converted by the application into a graph-based representation. The landmarks and their connections with each other must have been entered previously by the user in a separate, time-consuming process. In the second step, the application utilizes the generated profile to simplify the route by incorporating a subset of the known landmarks into the route as intermediate points that the user can reach without further assistance. The driving instructions are then adjusted to this route, which might increase the driving distance significantly if the familiar landmarks are far-off from the original route; in some cases, the route might even be changed completely. This means that a trade-off is made between the simplicity of driving instructions, and the economy of the chosen route (e.g. in terms of time and fuel cost). This might be applicable, if the alternative route is only a minor detour. Yet, we think that the routes that have been optimized in many ways should not be modified. Instead of recalculating the entire path, we propose to incorporate the driver's prior spatial knowledge to provide individual directions.

The approach proposed by Richter et al. [14] assumes that the user is familiar with any given area. Should the driver need more detailed information on the current part of the route, that information has to be requested explicitly in a dialogue with the system. This requires regular, and possibly constant, interaction with the system while driving, which entails the danger of frequent distraction and therefore has to be viewed critically.

Finally, it should be mentioned that switching off the navigation system while driving in a familiar area is usually not desired, because the system can still provide useful extra information, for example on the current traffic situation or diversion routes. and also avoids the user from unnecessary interactions while driving.

\section{ABSTRACTING AND ENRICHING ROUT- ING INSTRUCTIONS}

Ideally, routing directions should correspond completely to the individual driver's knowledge. Such knowledge would include familiar areas and routes, but also places or objects that are well-known either to the individual or to the general public, such as landmarks, the homes of close friends and relatives and many other spatially referenceable elements. To date, however, no complete or consistent models of the full range of such information are available. Our approach is therefore to extract and integrate as much routing-related information as possible from existing services and to enrich and filter it in order to provide directions at different levels of detail . The method should allow to switch between different LOD using destination descriptions in well-known areas ("Drive towards Cologne"), switching to detailed turnby-turn directives when driving through unknown regions. Instructions should be summarized according to the principle of chunking where appropriate. 
In accordance with these requirements, we now introduce a method to convert conventional directions into sets of instructions of varying granularity for a given start and destination address. Standard route descriptions (such as those provided by Google Maps and similar services) are divided into several steps, each one with defined waypoints for the start and end point. We convert these routes into a layered description model as depicted in Figure 1.

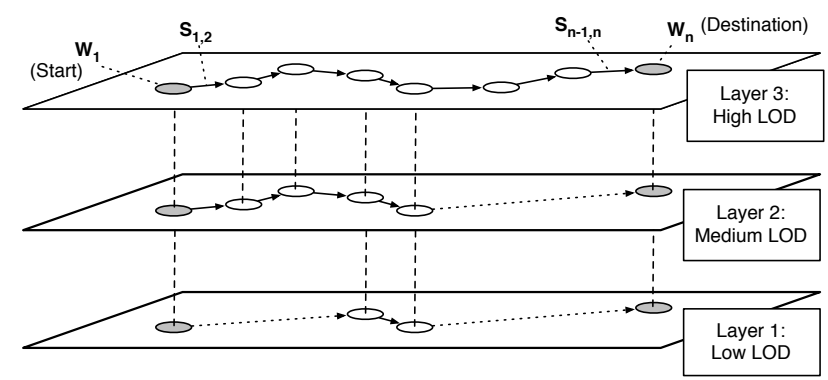

Figure 1: A route with $\boldsymbol{n}$ waypoints $W_{i}$ and $\boldsymbol{n}-1$ steps $S_{i, j}$ converted into 3 layers of detail. Dashed lines mark the $W_{i}$ present in all layers, dotted arrows mark merged instructions that have to be created.

While the highest layer contains the original set of instructions, the lower ones contain successively less detailed information, using the type of waypoint as a filter. The method comprises the following steps.

1. Request a conventional route description using a vendor API such as those provided by Google or Bing.

2. Enrich the waypoints of that route with semantic data.

3 . For $k$ desired LODs, create $k$ layers, each one containing only those waypoints that are supposed to be displayed within this layer.

4. Generate summarized directions for merged route segments (chunking).

\section{Step 1: Requesting directions}

Directions can be obtained from various sources and vendors such as Bing or Google. We use the Google Directions API ${ }^{1}$ to request a route description for a given start and destination address. Generally, any other source can be integrated with moderate implementation effort. Listing 1 illustrates an excerpt of such a route description.

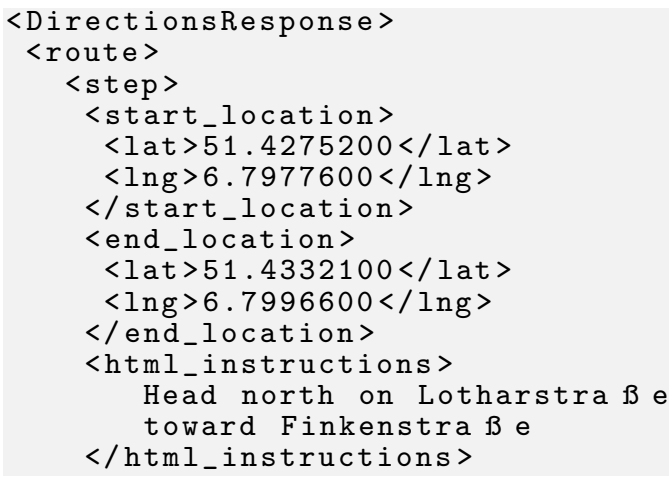

${ }^{1}$ http://code.google.com/apis/maps

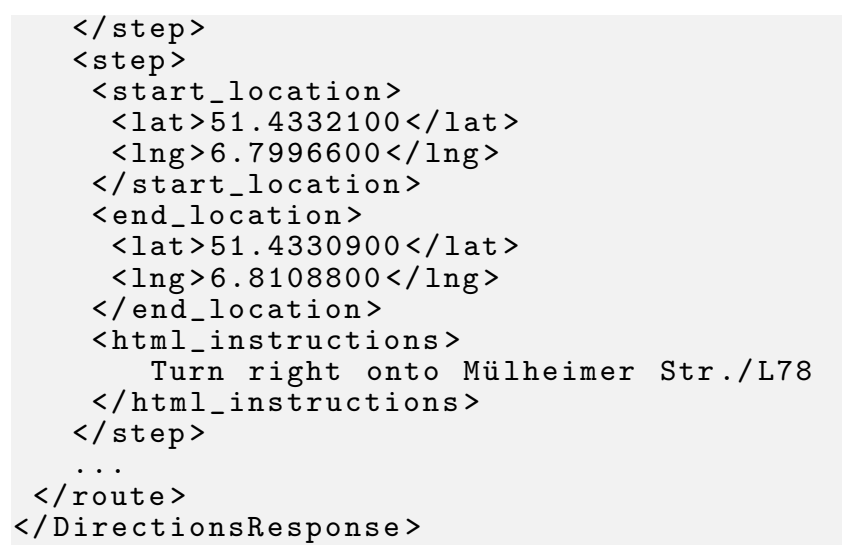

Listing 1: Excerpt from a route description obtained from the Google Directions API.

To provide means to abstract from this source code level we now introduce some concepts that are used throughout the rest of this article. As illustrated in Figure 1 we define a route as a directed graph $R=\{W, S\}$ containing $n$ waypoints $W_{1}, \ldots, W_{n}$ (the vertices) and steps $S_{i, j}$ (directed edges from $W_{i}$ to $W_{j}$ ). We denote $W_{1}$ as the starting point of the route and $W_{n}$ as its final destination. The original route contains exactly the steps from each waypoint $W_{i}$ to its respective successor $W_{i+1}$ for all $i<n$.

\section{Step 2: Adding semantics}

After obtaining the structured route information, we enrich the waypoints with semantic information obtained from geoservices. We therefore transform the route description into a semantic model, which allows us to characterize each waypoint on the basis of its properties comprehensively. The use of semantic models has several advantages:

- Standardization: As information comes from various sources, each with their own formats and specifications, we need a standard to cover all these kinds of information. Semantic models are flexible enough to import all information provided by the original sources and make them accessible in a unified way using Semantic Web languages.

- Extensibility: Standardization also enables the integration of new information sources as well, regardless of their format.

- Ease of data processing: If the models are encoded in a standardized language such as RDF or OWL, they can be queried using the SPARQL Protocol and $R D F$ Query Language (SPARQL).

- Additional services: The use of standardized semantic models lays the groundwork for future integration of services other than those directly related to navigation. Recommender systems that incorporate semantic data [6] could, for instance, find filling stations with attractive bargains or popular restaurants on the way.

In addition to the general information of an itinerary, such as location coordinates, street names and driving instructions, a semantic description includes further information, such as a classification of each route point based on its geographical 
or other properties. This means that a place can either be characterized as town, city, park, etc. and the connection between two places as a street, road or motorway. This hierarchical distinction later enables filtering to distinguish the different LODs.. Figure 2 shows such a semantic route representation enhanced with additional information.

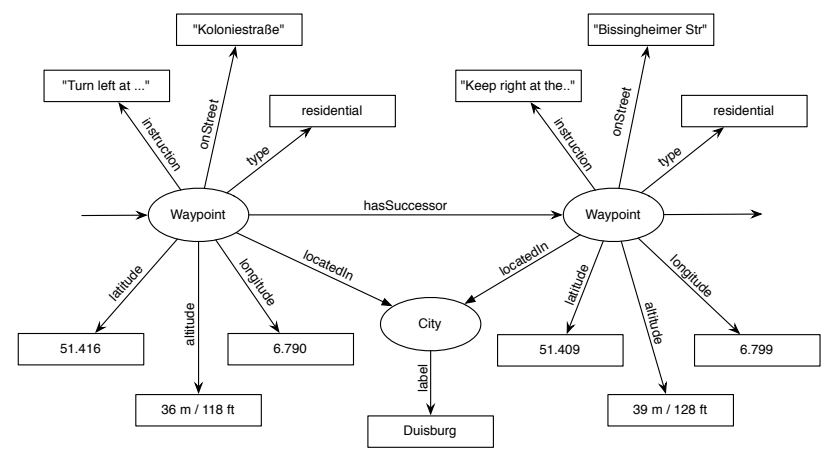

Figure 2: Part of a route description enriched with semantic information.

To semantically enrich the original route description, a variety of services can be used in principle, all of which providing comprehensive background knowledge related to spatial features of the ways, structures and landscapes around the waypoints of an itinerary [2]. We chose GeoNames ${ }^{2}$ and Google Geocoding $\mathrm{API}^{3}$ due to performance reasons and licensing costs, but other services such as LinkedGeoData ${ }^{4}$, or Topocoding ${ }^{5}$ could have been used as well.

\section{Step 3: Creating layers}

Based on the added semantics, we can now apply SPARQL filters such as the one illustrated in Listing 2 to select only those waypoints meeting certain criteria (in this case, the street type of the waypoint should be greater than "primary" according to the hierarchy used by GeoNames). Alternatively it is also possible to filter by other criteria, for instance by cities with a population greater than 100.000. Generally, any waypoint property can be used for filtering.

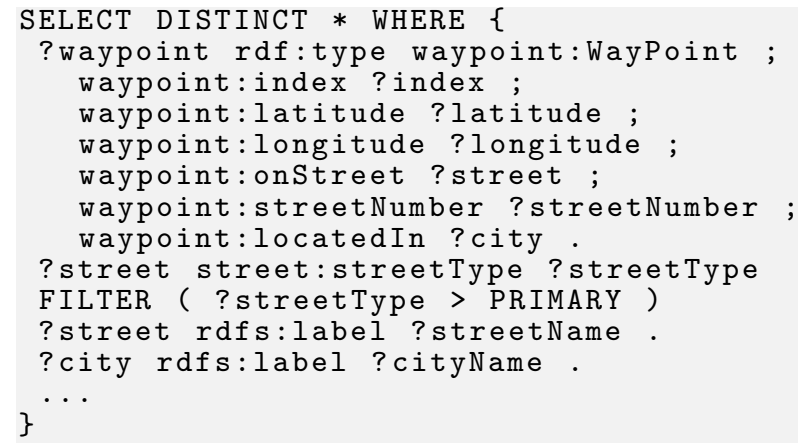

Listing 2: A SPARQL filter: Select all waypoints that belong to a higher category than 'primary' and

\footnotetext{
${ }^{2}$ http://www.geonames.org

${ }^{3}$ http://code.google.com/apis/maps

${ }^{4}$ http://linkedgeodata.org

${ }^{5}$ http: //www.topocoding.com
}

also request a number of attributes such as latitude longitude etc.

We can now specify the number of distinct granularity levels as well as SPARQL-filters that select only those waypoints appropriate for the particular layer. The waypoints for start and destination are always included and each layer $L_{k}$ automatically includes at least all waypoints from layer $L_{k-1}$. The different route descriptions constitute a layered model, where each layer represents a description on a certain LOD: $L_{1}$ is the most abstract layer, whereas $L_{m}$ includes all waypoints of the original description $(1<k<m)$ (Figure 1 illustrates a layered model with $m=3$ ). We denote waypoints that are both in $L_{i}$ and $L_{j}$ as synchronization points between $L_{i}$ and $L_{j}$. They are later used to combine segments from different layers to a personalized set of directions. Synchronization points at the abstract layer $L_{1}$ (and thus also present at each other layer) are called global synchronization points.

\section{Step 4: Summarizing directions}

Due to the filtering process, a certain number of waypoints are omitted in each layer except the highest $\left(L_{m}\right)$. As a result, $W_{1}$ might for instance directly be followed by $W_{4}$, entailing the need to generate a direction for step $S_{1,4}$ as the original directions only cover segments for consecutive waypoints. We used the functions $\operatorname{city}\left(W_{i}\right)$ and $\operatorname{street}\left(W_{i}\right)$ to obtain the city and street names, respectively, from the enriched model. We then apply a set of linguistic rules to generate directions $S_{i, j}$ for arbitrary $i, j \mid 1 \leq i<j \leq n$. Listing 3 shows one of those rules in a simplified fashion.

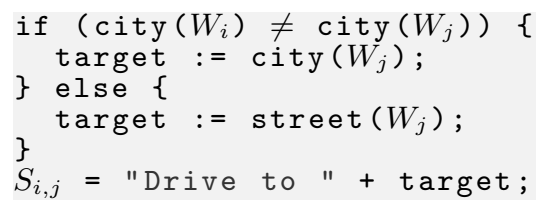

Listing 3: If $W_{i}$ and $W_{j}$ are within different cities the simpified direction is to drive to that city. If not drive to the street of $W_{j}$.

The following example illustrates the result. A route from $W_{1}$ to $W_{9}$ can be described in three different levels of detail:

\section{Level 3 (Original description):}

1. Head north on Lotharstraße toward Finkenstraße $\left(S_{1,2}\right)$

2. Turn right onto Mülheimer Str./L78 $\left(S_{2,3}\right)$

3. Exit on the left onto Carl-Benz-Straße/L138 $\left(S_{3,4}\right)$

4. Slight right to merge onto A40 toward Essen $\left(S_{4,5}\right)$

5. Take exit 19-Mülheim a. d. Ruhr-Heißen toward MHHeißen $\left(S_{5,6}\right)$

6. Turn left onto Frohnhauser Weg/L132 (signs for EFrohnhausen/Alexanderstraße). Continue to follow Frohnhauser Weg $\left(S_{6,7}\right)$

7. Turn left onto Reuterstraße $\left(S_{7,8}\right)$

8. Take the 1st left onto Inselstraße. Destination will be on the right $\left(S_{8,9}\right)$

Level 2: 
1. Head north on Lotharstraße toward Finkenstraße $\left(S_{1,2}\right)$

2. Turn right onto Mülheimer Str./L78 $\left(S_{2,3}\right)$

3. Exit on the left onto Carl-Benz-Straße/L138 $\left(S_{3,4}\right)$

4. Slight right to merge onto A40 toward Essen $\left(S_{4,5}\right)$

5. Take exit 19-Mülheim a. d. Ruhr-Heißen toward MHHeißen $\left(S_{5,6}\right)$

6. Drive to Inselstraße $\left(S_{6,9}\right)$

\section{Level 1:}

1. Drive to $\mathrm{A} 40\left(S_{1,4}\right)$

2. Slight right to merge onto A40 toward Essen $\left(S_{4,5}\right)$

3. Take exit 19-Mülheim a. d. Ruhr-Heißen toward MHHeißen $\left(S_{5,6}\right)$

4. Drive to Inselstraße $\left(S_{6,9}\right)$

Table 1 shows an exemplary overview of the generated directions for the route start section.

\begin{tabular}{|c|c|c|}
\hline Layer 1 & Layer 2 & Layer 3 \\
\hline \multirow{3}{*}{$S_{1,4}$} & \multicolumn{2}{|c|}{$S_{1,2}$} \\
\cline { 2 - 3 } & \multicolumn{2}{|c|}{$S_{2,3}$} \\
\cline { 2 - 3 } & \multicolumn{2}{|c|}{$S_{3,4}$} \\
\hline \multicolumn{3}{|c|}{$S_{4,5}$} \\
\hline \multicolumn{2}{|c|}{$S_{5,6}$} \\
\hline \multirow{3}{*}{$S_{6,9}$} & $S_{6,7}$ \\
\cline { 2 - 2 } & $S_{7,8}$ \\
\cline { 2 - 2 } & $S_{8,9}$ \\
\hline
\end{tabular}

Table 1: Exemplary overview of the generated directions for the route start section.

\section{Implementation}

We implemented the functionality described in Section 3 in a Java library called LayerGenerator, which is part of our $M I A^{6}$ library collection. It can be embedded into Java-based web, desktop, or mobile applications.

Programs using the LayerGenerator have to configure a number of layers and can then simply pass a start and destination address as if they were using Google Maps. The LayerGenerator then produces a LayerModel object that can be used as a business object within the application.

The layer model does not provide means for sensing or maintaining user profiles. It was designed and implemented as a one-purpose library, following UNIX-principles [12] such as the Rule of Modularity: Write simple parts connected by clean interfaces. So, a program using the LayerGenerator has to find out the appropriate LOD itself ${ }^{7}$. It then can use a layered model in order to display a set of instructions according to the driver's knowledge and preferences.

\section{EMPIRICAL EVALUATIONS}

We conducted two studies to assess users' preferences for different LODs in routing instructions and to evaluate the ap-

${ }^{6} \mathrm{MIA}$ is an acronym for Mobile Intelligent Assistant. It includes several libraries for mobile enhancements. Other libraries are, however, out of the scope of this article

${ }^{7}$ However, we are developing other MIA libraries that aim at exactly that goal. propriateness of the directions generated by our system. The studies were designed to take into account that the system, while being functional, was not yet integrated with a real navigation system. The first one was a pre-study, designed as an in-car "partial" Wiz-of-Oz study where users received instructions from the experimenter while driving. 19 persons participated in this study, receiving directions with varying LODs that were generated beforehand by the system. The second study was designed as an online experiment with 118 participants in which stationary users were presented with system-generated personalized directions. The goal of the two studies was to examine the following hypotheses (H3 was only addressed in the second study):

H1: The preferred LOD depends on the route section.

H2: The preferred LOD depends on the participant's knowledge of the particular area.

H3: Users prefer instructions generated by the LayerGenerator over those generated by Google Maps.

\section{Road Study}

In this pre-study, we examined which LOD was requested by drivers for partially known routes and whether the instructions generated by the system were at all sufficient to reach to target. 19 persons participated (12 female, 7 male; average age 41.36 with a standard deviation of 16.55). The task was to head for destinations between 20 and $50 \mathrm{~km}$ away from the respective starting point. Each route was subdivided into a start, transition and destination section, where we used the global synchronization points for partitioning the routes. We chose the itineraries in a way that all participants were familiar with either the respective start or destination area.

Route directions were generated beforehand based on the starting or end points familiar to the driver. However, instead of controlling and presenting the instructions by a real navigation system, a fellow passenger acted as an instructor reading out the pre-calculated directions. The instructions started by first presenting abstract (Layer 1) driving directives. If the driver did not consider them sufficient, the instructor gradually increased the LOD for the section upon request and also decreased it again when not needed by the driver. The driver's knowledge of the area was determined in dialogue by directly asking them how they estimate their knowledge of the area.

\section{Results}

It showed that different LOD were requested in the three sections of the drive, depending on the level of knowledge (Figure 3). As hypothesized, participants with low knowledge needed detailed instructions, whereas in all sections more abstract instructions were sufficient when knowledge was high.

\section{Online Study}

In a second step, we conducted an online study in order to determine users' preference of LOD for arbitrary, but partially known routes. 118 participants aged 17 to 65 years participated in the survey. Participants came from Germany and other European countries. About one quarter $(n=37)$ was female and three quarters $(n=81)$ were male. Partic- 


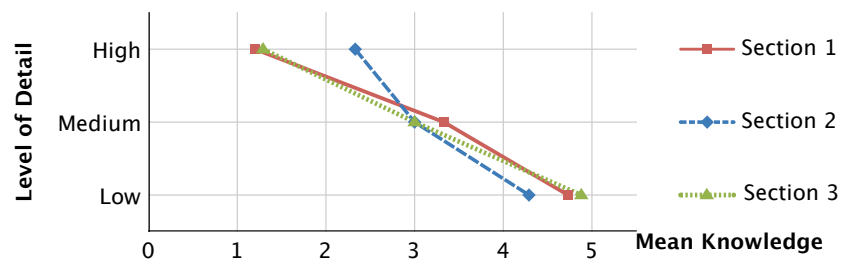

Figure 3: With higher knowledge (x-axis), the participants needed less details (y-axis). This was true throughout all sections.

ipants were acquired via social networks and other Internet platforms.

In a specially designed web application based upon the LayerGenerator, participants first had to enter a geographical area they were familiar with. ${ }^{8}$ Then, a route was generated, where the familiar area was randomly used either as the route's start or end point. The unknown end of the route was always chosen at random. The length of the generated routes was varied systematically between short (approx. 25 $\mathrm{km}$ ) and long (approx. $75 \mathrm{~km}$ ), resulting in four different types of routes.

Upon creating a route, directions were generated and presented on a web page. We automatically partitioned each route into three sections (start, middle, end) based upon the global synchronization points. We considered the section containing the provided address as very familiar, the transition section (often a major highway) as partly familiar, and the randomly chosen address as unfamiliar.

\section{Task 1: Rate the instructions for each route section}

Participants were sequentially presented with instructions for the start, middle and end section of a route. For each section, three variants of instruction sets with different LOD (high, medium, low) were shown simultaneously on the web page (Figure 4). Participants had to rate each instruction set on a scale from 1 to 5 (dependent variable) and in addition were asked to state their knowledge of the respective section, also on a 1-5 scale.

\section{Task 2: Compare personalized route descriptions to Google Maps directions}

In the second task, the participants were presented with a personalized description of a complete route generated by LayerGenerator, as well as the corresponding conventional route description produced by Google Maps. The route itself was the same as in the first task. This time, however, a participant's knowledge of the different sections was taken into account, using the familiarity ratings given in task 1 . We generated a completely personalized route description by concatenating the descriptions best corresponding to the stated knowledge level for each section. A high knowledge level resulted in a low LOD, medium knowledge in medium LOD, and low knowledge in high LOD. The participants were asked to compare and rate the personalized route description to the original route from Google Maps using a

\footnotetext{
8 i. e. a street address and city plus postal code.
}
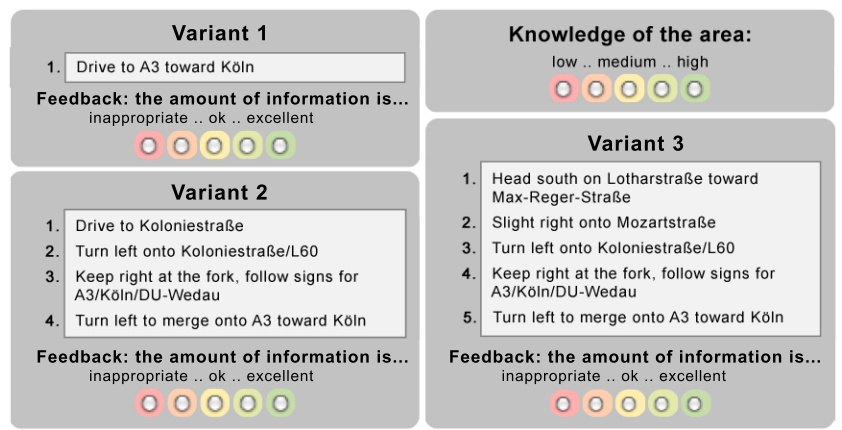

Figure 4: In the first task the participants were shown descriptions on different LODs for each route section. These were evaluated as a function of their spatial knowledge.

Likert-scale $(1=$ very unsuitable to $5=$ very suitable).

\section{Task 3: Arranging personalized instructions}

In this task, we asked participants to manually adjust the LOD for each route section so that the amount of information would be just sufficient. Starting with the highest LOD, the amount of information shown for each route section could be adjusted by pressing 'plus' and 'minus' buttons (Figure 5). The route presented in this part was different from the one in the first two tasks, but was built using the same criteria (i.e. placing either the start or destination in the familiar area that was initially entered by the user, systematically varying between short and long routes).
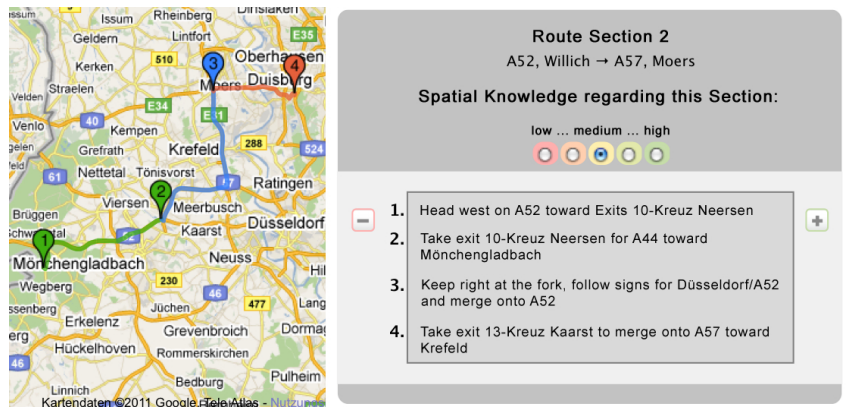

Figure 5: In the last task the participants could set each section to the desired level of abstraction.

\section{Results for Task 1}

To analyze how the ratings of the LODs differed between the three route sections, depending on the spatial knowledge of the participants, we conducted a repeated measures analysis of covariance (ANCOVA). The ratings for the three LOD in each of the three sections served as dependent variables and the participants' spatial knowledge as covariate.

The ratings of the LOD differed significantly in all three sections and interacted with driver's knowledge (Table 2). The effects were stronger in section 1 and 3 and weaker in section 2. In section 1 and 3 , higher knowledge of a section and a low LOD resulted in a higher rating, whereas a lower rating could be observed when the participant's knowledge was also low. 


\begin{tabular}{ccccccc} 
Sec. & ISE & $\mathbf{F}$ & $\mathbf{p}$ & $\mathbf{d f 1}$ & $\mathbf{d f 2}$ & $\boldsymbol{\eta}^{\mathbf{2}}$ \\
\hline \hline \multirow{2}{*}{1} & LOD & 255.67 & $\leq 0.001$ & 1.65 & 191.30 & 0.69 \\
& LOD $\times$ K & 160.44 & $\leq 0.001$ & 1.65 & 191.30 & 0.58 \\
\hline \multirow{2}{*}{2} & LOD & 10.39 & $\leq 0.001$ & 2.00 & 232.00 & 0.08 \\
& LOD $\times$ K & 3.73 & $\leq 0.05$ & 2.00 & 232.00 & 0.03 \\
\hline \multirow{2}{*}{3} & LOD & 71.23 & $\leq 0.001$ & 1.43 & 165.76 & 0.38 \\
& LOD $\times$ K & 88.58 & $\leq 0.001$ & 1.43 & 165.76 & 0.43 \\
\hline
\end{tabular}

Table 2: Results per section for the inner subject effects (ISE) and the interaction between the levelof-detail (LOD) and the participants' spatial knowledge $(\mathrm{K}) \cdot \eta^{2}$ shows the effect size $(\mathrm{F}=$ test-statistics; $\mathrm{p}=$ significance; $\mathrm{df} 1, \mathrm{df} 2=$ degrees of freedom $)$. The ratings were significantly different depending on the LOD and interacted with the knowledge of the participants of the corresponding section.

To analyze how the ratings differed depending on the knowledge of the participant, we analyzed the parameter estimates for knowledge as covariate (Tables 3 to 5). A linear trend could be observed: a high LOD with high knowledge resulted in a worse rating and a high LOD with low knowledge in a better rating, respectively (Figure 7).

\begin{tabular}{lccccc} 
LOD & Parameter & $\mathbf{b}$ & $\mathbf{T}$ & $\mathbf{p}$ & $\boldsymbol{\eta}^{2}$ \\
\hline \hline \multirow{2}{*}{ High } & $\mathrm{C}$ & 4.98 & 33.30 & $\leq 0.001$ & 0.91 \\
& Knowledge & -0.50 & -10.19 & $\leq 0.001$ & 0.47 \\
\hline \multirow{2}{*}{ Medium } & $\mathrm{C}$ & 2.48 & 18.80 & $\leq 0.001$ & 0.75 \\
& Knowledge & 0.17 & 3.92 & $\leq 0.05$ & 0.12 \\
\hline \multirow{2}{*}{ Low } & $\mathrm{C}$ & 0.38 & 2.70 & $\leq 0.01$ & 0.06 \\
& Knowledge & 0.70 & 15.22 & $\leq 0.001$ & 0.67 \\
\hline
\end{tabular}

Table 3: Parameter estimates for the covariate spatial knowledge for the LODs in the first section $(C=$ constant; $b=$ regression coefficient; $T=$ teststatistics; $p=$ significance; $\eta^{2}=$ effect Size). The spatial knowledge had a medium to high effect on the ratings of high and low LODs. An increase of spatial knowledge by 1 point, for instance, lead to a decreased rating of the highly-detailed instructions by -0.5 points.

\begin{tabular}{lccccc} 
LOD & Parameter & $\mathbf{b}$ & $\mathbf{T}$ & $\mathbf{p}$ & $\boldsymbol{\eta}^{2}$ \\
\hline \hline \multirow{2}{*}{ High } & $\mathrm{C}$ & 3.51 & 13.65 & $\leq 0.001$ & 0.62 \\
& Knowledge & 0.12 & 1.57 & n.s. & 0.02 \\
\hline \multirow{2}{*}{ Medium } & $\mathrm{C}$ & 4.01 & 14.09 & $\leq 0.001$ & 0.63 \\
& Knowledge & 0.06 & 0.72 & n.s. & 0.00 \\
\hline \multirow{2}{*}{ Low } & $\mathrm{C}$ & 3.12 & 11.96 & $\leq 0.001$ & 0.55 \\
& Knowledge & 0.23 & 2.81 & $\leq 0.01$ & 0.06 \\
\hline
\end{tabular}

Table 4: Parameter estimates for section 2. Only in the low LOD condition did the effect of spatial knowledge have a significant influence on the rating, but with a rather small effect size $\left(\eta^{2}=0.06\right)$.

Results for Task 2

As shown in Figure 6, the personalized routes generated by the LayerGenerator were rated with 4.47 points (SD 0.83),

\begin{tabular}{lccccc} 
LOD & Parameter & $\mathbf{b}$ & $\mathbf{T}$ & $\mathbf{p}$ & $\boldsymbol{\eta}^{2}$ \\
\hline \hline \multirow{2}{*}{ High } & $\mathrm{C}$ & 4.95 & 18.85 & $\leq 0.001$ & 0.75 \\
& Knowledge & -0.50 & -7.60 & $\leq 0.001$ & 0.33 \\
\hline \multirow{2}{*}{ Medium } & $\mathrm{C}$ & 3.24 & 15.43 & $\leq 0.001$ & 0.67 \\
& Knowledge & 0.01 & 0.23 & n.s. & 0.00 \\
\hline \multirow{2}{*}{ Low } & $\mathrm{C}$ & 0.54 & 1.98 & $\leq 0.05$ & 0.03 \\
& Knowledge & 0.73 & 10.89 & $\leq 0.01$ & 0.51 \\
\hline
\end{tabular}

Table 5: Parameter estimates for section 3. Similar to section 1, spatial knowledge had a medium to high effect on the ratings of high and low LODs. An increase of spatial knowledge by 1 point lead to an increased rating of the low-detail instructions by 0.73 points.

which is significantly better than the unpersonalized routes generated by Google Maps. The routes generated by Google Maps were rated with 2.80 points (SD 1.17) on average $(t(117)=11.89, \leq 0.001)$.

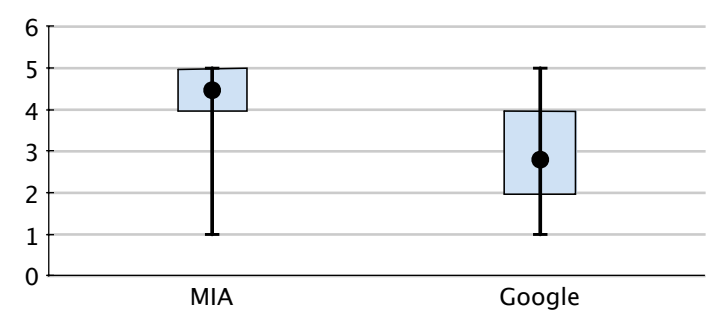

Figure 6: The LayerGenerator was rated significantly better than Google Maps (4.47 vs. 2.80 on average). The boxes show the standard deviation and the whiskers the maxima and minima. As the standard deviation shows, the ratings of the routes generated by the LayerGenerator were mostly between four and five points.

\section{Results for Task 3}

Depending on the individual spatial knowledge, the chosen LOD differed significantly in all sections (Figure 7). Participants with lower spatial knowledge of a section chose a higher LOD, and chose a lower LOD with increasing knowledge. The effect is weaker for the medium LOD than for the high or low LOD.

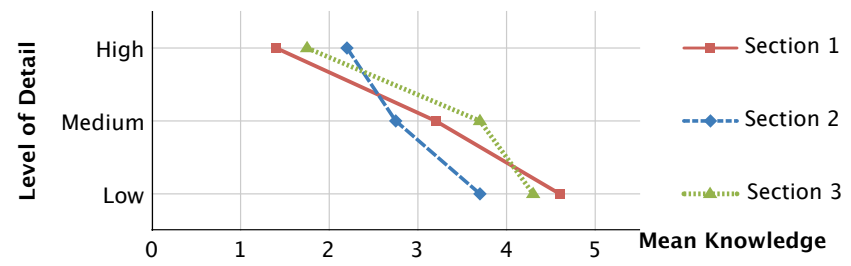

Figure 7: In addition to the decreasing need for details (see the road study), the participants also preferred less details with increasing knowledge, especially in section 1 and 3 . 


\section{DISCUSSION AND OUTLOOK}

The results of the two studies support our hypotheses that drivers prefer different levels of detail depending on their knowledge of the area. Furthermore, it could be shown that our approach of semantically enriching and filtering information extracted from existing services can provide meaningful and effective instructions for the driver. The online study carried out corroborates our assumption that the personalized directions produced by this techniques are appropriate for reaching the intended destination and are preferred by users. In particular, the descriptions generated by MIA have been rated significantly better compared to those provided by Google Maps. The concept of producing abstractions by spatial chunking of waypoints has been evaluated positively, which is in tune with our expectations as well as with the corresponding literature. Regarding personalization for different route sections, the empirical data provide evidence that personalization is especially meaningful in the start and destination areas of a route. In the middle section, personalization often is not meaningful or even possible as this section often includes long drives on a motorway without many changes of direction.

While the results obtained are promising, the method presented is clearly just a first step towards fully adaptive and personalized navigation. As yet we have not realized a method for automatically determining the driver's knowledge of a route or some specific geospatial waypoint. However, the approach of semantically enriching route descriptions opens a range of possibilities for integrating machine learning techniques into the system. Based on a driver's actual route data, frequently used roads or places visited could feed into mechanisms for acquiring a driver model representing geospatial knowledge or driving preferences at an appropriate semantic level.

Furthermore, we have started first promising experiments with descriptions exported from the location-based social networking service Foursquare. However, since sites such as Foursquare do not track the user's whereabouts permanently, this solution seems only appropriate in combination with other techniques. Also, privacy aspects have to be considered that may prohibit total user tracking.

\section{REFERENCES}

[1] A. Al Mahmud, O. Mubin, and S. Shahid. User experience with in-car gps navigation systems: Comparing the young and elderly drivers. In MobileHCI '09: Proceedings of the 11th International Conference on Human-Computer Interaction with Mobile Devices and Services, pages 90:1-90:2, 2009.

[2] S. Auer, J. Lehmann, and S. Hellmann. Linkedgeodata: Adding a spatial dimension to the web of data. In ISWC '09: The 8th International Semantic Web Conference, volume 5823 of Lecture Notes in Computer Science, pages 731-746. Springer, 2009.

[3] M. Bettitt, G. Burnett, and A. Stevens. Defining driver distraction. In Proceedings of the World Congress on Intelligent Transport Systems, 2005.

[4] J. Forlizzi, W. C. Barley, and T. Seder. Where should i turn: Moving from individual to collaborative navigation strategies to inform the interaction design of future navigation systems. In CHI '10: Proceedings of the 28th International Conference on Human Factors in Computing Systems, pages 1261-1270, New York, NY, USA, 2010. ACM.

[5] J. Hu, I. Kaparias, and M. G. Bell. Spatial econometrics models for congestion prediction with in-vehicle route guidance. IET Intelligent Transport Systems, 3(2):159-167, 2009.

[6] T. Hussein, T. Linder, W. Gaulke, and J. Ziegler. Context-aware recommendations on rails. In CARS , 09: Proceedings of the 1st Workshop on Context-Aware Recommender Systems, New York, NY, USA, 2009.

[7] A. Klippel, S. Hansen, K.-F. Richter, and S. Winter. Urban granularities - a data structure for cognitively ergonomic route directions. GeoInformatica, 13(2):223-247, 2009.

[8] A. Klippel, H. Tappe, and C. Habel. Pictorial representations of routes: Chunking route segments during comprehension. In C. Freksa, W. Brauer, C. H. Christopher, and K. F. Wender, editors, Spatial cognition III, number 2685 in Lecture Notes in Artificial Intelligence, pages 11-33. Springer, 2003.

[9] T. Kujala. Efficiency of visual time-sharing behavior: The effects of menu structure on poi search tasks while driving. In AutomotiveUI '09: Proceedings of the 1st International Conference on Automotive User Interfaces and Interactive Vehicular Applications, pages 63-70, New York, NY, USA, 2009. ACM.

[10] K. Lovelace, M. Hegarty, and D. Montello. Elements of good route directions in familiar and unfamiliar environments. In C. Freksa and D. Mark, editors, Spatial Information Theory. Cognitive and Computational Foundations of Geographic Information Science, number 1661 in Lecture Notes in Computer Science, pages 65-82. Springer, 1999.

[11] K. Patel, M. Y. Chen, I. Smith, and J. A. Landay. Personalizing routes. In UIST '06: Proceedings of the 19th Annual ACM Symposium on User Interface Software and Technology, pages 187-190. ACM, 2006.

[12] E. S. Raymond. The Art of UNIX Programming. Eddison-Wesley, 2003.

[13] K. Richter. From turn-by-turn directions to overview information on the way to take. Location based services and telecartography, pages 205-216, 2007.

[14] K.-F. Richter, M. Tomko, and S. Winter. A dialog-driven process of generating route directions. Computers, Environment and Urban Systems, 32(3):233-245, 2008.

[15] A. Stevens and P. Coupe. Distortions in judged spatial relations. Cognitive Psychology, 10(4):422-437, 1978.

[16] M. Tomko. Destination Descriptions in Urban Environments. PhD thesis, University of Melbourne, 2007.

[17] B. Tversky. Spatial Information Theory: A Theoretical Basis for GIS, volume 716 of Lecture Notes in Computer Science, chapter Cognitive Maps, Cognitive Collages and Spatial Mental Models, pages 14-24. Springer, Berlin, Germany, 1993.

[18] J. Yoon, B. Noble, and M. Liu. Surface street traffic estimation. In MobiSys '07: Proceedings of the 5th International Conference on Mobile Systems, Applications and Services, pages 220-232, New York, NY, USA, 2007. ACM. 